\title{
Delayed Hypersensitivity Reaction to Liraglutide: A Case Report
}

Carvallo $\mathrm{A}^{1}$, Silva $\mathrm{C}^{2}$, Gastaminza G $\mathrm{G}^{1,3}$, D'Amelio $\mathrm{CM}^{1,3}$

${ }^{1}$ Department of Allergy and Clinical Immunology, Clínica Universidad de Navarra, Pamplona, Spain

${ }^{2}$ Department of Endocrinology and Nutrition. Clínica Universidad de Navarra, Pamplona, Spain

${ }^{3}$ Spanish Research Network on Allergy RD 1600060031 (ARADyAL: Red Nacional de Alergia-Asma, Reacciones Adversas y Alérgicas) of the Carlos III Health Institute, Madrid, Spain

J Investig Allergol Clin Immunol 2020; Vol. 30(5): 367-369 doi: 10.18176/jiaci.0521

Key words: Drug allergy. Liraglutide. GLP-1 receptor agonist. Delayed hypersensitivity.

Palabras clave: Alergia a medicamentos. Liraglutida. Agonista del receptor GLP-1. Hipersensibilidad retardada.

Glucagon-like peptide-1 receptor agonists (GLP-1RAs) are a class of drugs used in the treatment of type 2 diabetes. Liraglutide is currently the only GLP-1RA approved by the United States Food and Drug Administration and European Medicines Agency for treatment of obesity [1]. Allergic reactions have been reported with the exendin-4-based subtype of GLP-1RAs, although no cases with new human GLP-1 analogues, such as liraglutide and semaglutide, have been confirmed by allergy tests to date [2-5].

We present the case of a 42-year-old woman diagnosed with obesity, for which she started treatment with liraglutide at $0.6 \mathrm{mg} / \mathrm{d}$, with weekly increments of $0.6 \mathrm{mg}$ until a maximum dose of $3 \mathrm{mg} / \mathrm{d}$ was achieved. One week after starting the daily 3-mg subcutaneous dose, she presented with pruriginous erythematous macules at the injection site, which appeared 24 hours after the injection. This pattern recurred for 4 days, after which her endocrinologist referred her to our allergy department. At the physical examination, she had 2 macules $(35 \times 20 \mathrm{~mm})$ on the lower left abdomen and a smaller one on the lower right abdomen at the injection sites. A skin prick test (SPT) performed with liraglutide $(6 \mathrm{mg} / \mathrm{mL})$ was negative. This was followed by an intradermal skin test (IDT), which was negative at the $1 / 100(0.06 \mathrm{mg} / \mathrm{mL})$ and $1 / 10(0.6 \mathrm{mg} / \mathrm{mL})$ dilutions; the $1 / 1(6 \mathrm{mg} / \mathrm{mL})$ dilution was initially negative, but clearly positive at the 24-hour reading $(22 \times 17 \mathrm{~mm})$ (Figure). The positive dilution was tested in 5 healthy control individuals who had not been exposed to the drug, with negative results at the immediate and delayed readings. The patient was diagnosed with delayed allergy to liraglutide, and the drug was discontinued. She received topical corticosteroids, with remission of the lesions without recurrence at the 2-week follow-up. The patient had no previous history of atopy. She had a normal blood eosinophil count and liver function test results. She had never previously taken GLP-1RAs. The study was completed with a patch test to 


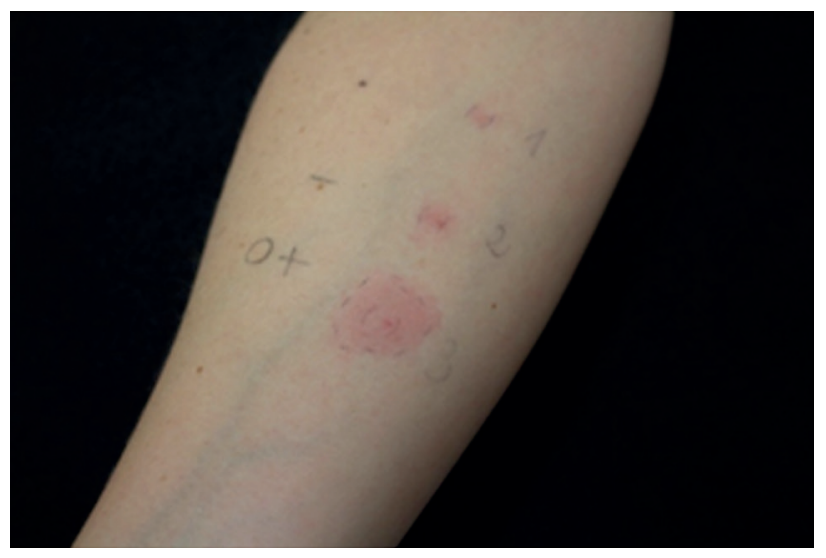

Figure. Intradermal skin test with liraglutide showing the positive 1/1 $(6 \mathrm{mg} / \mathrm{mL})$ dilution at the delayed 24 -hour reading.

liraglutide $(6 \mathrm{mg} / \mathrm{mL}$, as is), which was negative at the 48 -hour and 96-hour readings. Semaglutide was suggested as an alternative to liraglutide. SPT $(1.34 \mathrm{mg} / \mathrm{mL})$ yielded negative results, as did IDT to this drug at $1 / 100,1 / 10$, and $1 / 1$ dilutions $(0.01 \mathrm{mg} /$ $\mathrm{mL}, 0.13 \mathrm{mg} / \mathrm{mL}$, and $1.34 \mathrm{mg} / \mathrm{mL}$, respectively).

Allergic reactions to the exendin-4-based subtype of GLP-1RAs have been described in the literature. Shamriz et al [2] reported a case of anaphylaxis after a first dose of lixisenatide, with an immediate positive IDT result to this drug and to exenatide, which the patient had received in the past. However, skin tests with liraglutide, which she had also received, were negative. The patient later tolerated the drug. The authors attributed this to the molecular differences between the 2 exendin-4-based drugs-exenatide and lixisenatide - and the human GLP-1 analogue, liraglutide. Homology between exendin-4-based GLP-1RAs and human GLP-1 is about $53 \%$, thus potentially explaining the patient's tolerance to liraglutide, which has $97 \%$ homology with human GLP-1 [6].

As for human GLP-1 analogues, Neel et al [3] reported a case of injection site reaction after 2 weeks of a daily 3-mg dose of liraglutide, which resolved after discontinuation of the drug; however, no allergy study was performed on this patient. Cogen et al [4] reported a case of exanthematous pustulosis in photoexposed areas after 2 days of liraglutide treatment, with resolution after several weeks of drug discontinuation and treatment with topical corticosteroids; no skin tests or photopatch tests were performed. More recently, Bovijn et al [5] reported a case of generalized erythematous plaques and nodules in a patient with peripheral blood eosinophilia who had started liraglutide treatment 2 weeks earlier. The lesions took 5 months to resolve after discontinuation of the drug and therapy with topical corticosteroids; no allergy study was conducted. To our knowledge, no cases of confirmed hypersensitivity reaction to liraglutide with positive skin test results have been reported to date.

The symptoms observed in the present case-pruriginous macules at the injection sites-were compatible with a delayed hypersensitivity reaction to liraglutide and resolved 2 weeks after discontinuation without residual skin lesions. In contrast with previously reported cases, no other associated skin lesions, such as pustules or nodules, were present. The diagnosis was confirmed with the positive IDT result. In order to rule out the possibility of an irritant reaction resulting from the concentration used, we tested the same dilution in 5 healthy controls and recorded negative results. In relation to the negative patch testing results, the sensitivity of this method in the diagnosis of systemic drug reactions appears to be dependent on the drug type, which would explain the results observed [7].

It is worth noting that skin tests to semaglutide were negative in the present case, even though the molecule is derived from liraglutide and both are analogues of human GLP-1. Differences between semaglutide and its precursor molecule include substitution of alanine with $\alpha$-aminoisobutyric acid at position 8 , substitution of lysine with arginine at position 34, and acylation of lysine with a stearic diacid at position 26 [8]. The fact that IDT was negative to semaglutide could suggest that the patient's sensitization was dependent on 1 or more of these changed molecular components. Consequently, semaglutide could be an alternative in patients allergic to liraglutide, despite the similarity between the 2 agents. In the present case, tolerance to semaglutide was not assessed.

We report a case of a delayed hypersensitivity reaction with positive skin test results to liraglutide. The negative results with semaglutide suggest that this drug could be used as an alternative in patients with allergy to liraglutide. Further studies are needed to establish a clear pattern of cross-reactivity between these 2 human GLP-1 analogues.

\section{Funding}

This work was supported by Instituto de Salud Carlos III (ISCIII) and cofunded by Fondo Europeo de Desarrollo Regional - FEDER for the Thematic Networks and Cooperative Research Centres: ARADyAL(RD16/0006/0031).

\section{Conflicts of Interest}

The authors declare that they have no conflicts of interest.

\section{References}

1. Isaacs D, Prasad Reddy L, Srivastava SB. Role of glucagon like peptide 1 receptor agonists in management of obesity. Am J Health Syst Pharm. 2016;73:1493-507.

2. Shamriz O, NaserEddin A, Mosenzon O, Sviri S, Tal Y. Allergic reaction to exenatide and lixisenatide but not to liraglutide: unveiling anaphylaxis to glucagon-like peptide 1 receptor agonists. Diabetes Care. 2019;42:e141-2.

3. Neel NF, Ghobara Y, Turkmani M. Liraglutide-induced injection site reaction. J Dermatol Dermatol Surg. 2019;23:97-8.

4. Cogen A, Desai K, Elder D, Rosenbach M. Acute photodistributed exanthematous pustulosis associated with liraglutide treatment. JAMA Dermatol. 2019;155:1198-200.

5. Bovijn L, Arianayagam S, Asher R. An unusual case of a generalised cutaneous drug reaction to liraglutide. Eur J Dermatol. 2019:29:675-6. 
6. Bode B. An overview of the pharmacokinetics, efficacy and safety of liraglutide. Diabetes Res Clin Pract. 2012;97:27-42.

7. Ohtoshi S, Kitami Y, Sueki H, Nakada T. Utility of patch testing for patients with drug eruption. Clin Exp Dermatol. 2014;39:279-83.

8. Lau J, Bloch P, Schaffer L, Petterson I, Spetzler J, Kofoed J et al. Discovery of the once-weekly glucagon-like peptide-1 (GLP-1) analogue semaglutide. J Med Chem. 2015;58:7370-80.

I Manuscript received February 4, 2020; accepted for publication March 27, 2020.

\section{Carmen D'Amelio}

Av. Pio XII, 36

Clínica Universidad de Navarra

31008 Pamplona, Spain

E-mail: cdamelio@unav.es 